\title{
INTERVENCIONES DE ORIENTACIÓN PARA EDIFICAR FORTALEZAS EN NIÑAS, NIÑOS Y ADOLESCENTES
} Counseling Interventions for Strengths Building in Children and Adolescents

Irma Arguedas-Negrini ${ }^{1}$

irma.arguedas@ucr.ac.cr
${ }^{1}$ Universidad de Costa Rica, Costa Rica

Fecha de recepción: 15/02/2020 Fecha de aprobación: 13/04/2020

\section{Resumen}

La edificación de fortalezas en niñas, niños y adolescentes es esencial para su desarrollo presente y futuro. Varios estudios realizados en diferentes países latinoamericanos coinciden con esta visión, en función de la prevención de obstáculos al desenvolvimiento, especialmente de poblaciones afectadas por condiciones de desigualdad. En este trabajo se discuten las formas en que los profesionales en Orientación pueden contrarrestar las dificultades que a menudo enfrenta la población estudiantil que se encuentra en condición de riesgo. Se parte de una perspectiva preventiva que recalca la conveniencia de atender las dificultades desde etapas tempranas. El propósito es enfatizar en la importancia de las intervenciones de Orientación, en la promoción del desarrollo del estudiantado, así como en su protección contra los efectos nocivos de la adversidad. Se realizó una revisión de la teoría acerca de las fortalezas humanas y la resiliencia, que sustenta las intervenciones de profesionales en Orientación para la edificación de las potencialidades de las personas orientadas. Se presentan los pilares de la resiliencia del estudiantado y las formas en que interaccionan. Además, se presenta información específica acerca de tres de las dificultades que con mayor frecuencia sufre el estudiantado de educación primaria y secundaria: dificultades académicas, problemas conductuales y situaciones familiares. Los conocimientos disponibles deben conectarse con la multiplicidad de acciones que implementan las instituciones educativas para el beneficio del alumnado.

Palabras clave: Educación primaria, Enseñanza secundaria, orientación, resiliencia.

\section{Abstract}

Strengths building is essential for boys' and girls' present and future development. Studies carried out in different Latin American countries agree that with this perspective, so that obstacles to development, especially of populations affected by conditions of inequality, are prevented. This paper discusses ways in which Guidance professionals can counteract the difficulties that the student population often faces, especially those who are at risk. It is based on a preventive perspective, which emphasizes the desirability of addressing difficulties from early stages. The purpose is to emphasize the importance of Guidance interventions, in the promotion of student development, as well as in their protection from the adverse effects of adversity. A review of the theory about human strengths and resilience was carried out, which underpins the relevance of the promotion of counselees' potentialities. The conditions for resilience and development, as well as ways in which they interact, are presented. Additionally, specific information about three of the difficulties that elementary and secondary school students more frequently face: academic difficulties, behavioral problems, and family situations, are discussed. The available knowledge must be connected with the multiplicity of actions that are implemented from the educational institutions for the benefit of their students.

Keywords: Elementary Schools, guidance, resilience, Secondary Education. 


\section{La institución educativa en su doble papel: promoción y protección}

Dentro de sus múltiples funciones, la escuela y el colegio juegan un papel trascendental como promotores del desarrollo integral y protectores contra los efectos nocivos de las adversidades que enfrentan niñas, niños y adolescentes (Masten et al., 2008). Desde sus comunidades educativas, las personas profesionales en Orientación contribuyen con la construcción de trayectorias vitales significativas y saludables en la totalidad del estudiantado, para lo cual es imperativo prestar especial atención a los estudiantes que, por diversas razones, se encuentran en condición de riesgo. Esto es congruente con Murillo et al. (2011), quienes promueven la atención a la diversidad, de forma que "todos los alumnos, independientemente de sus características o necesidades permanentes o temporales, han de enfrentarse a tareas estimulantes, que promuevan todo su potencial” (p. 15).

Es indiscutible que atender las dificultades en las primeras etapas es lo más conveniente, ya que como lo plantean Yates et al. (2008), es más factible corregir descarrilamientos pequeños cuando la persona tiene una trayectoria de desarrollo mayoritariamente positiva, que reencauzar a una joven o un joven que ya ha tomado rutas improductivas o riesgosas.

Se trata, según Masten (2011), de poner en marcha cascadas positivas en las que los logros y el fortalecimiento se alimentan mutuamente. Así como se presentan riesgos acumulados en los procesos de desarrollo, las comunidades, las instituciones y otros recursos pueden contribuir con la edificación de protecciones múltiples que se sostengan en el tiempo.

Lo anterior reviste especial importancia en nińas, niños y jóvenes que, debido a la desigualdad, sufren más cantidad de situaciones que perjudican su rendimiento, tales como los trastornos de conducta (González-Arratia et al., 2009).

\subsection{La Orientación centrada en fortalezas}

Smith (2006) define fortalezas como las capacidades y recursos que ayudan a una persona a superar o mitigar los obstáculos que se presentan en su vida, hacerla satisfactoria para sí misma y para los demás, y encontrarle un propósito que brinde un sentido de conexión con el mundo. Son formas de procesar información, de vivenciar situaciones y de realizar cambios. No son rasgos fijos, sino el producto de procesos dinámicos, contextuales y enraizados en la cultura. Las fortalezas son modificables; se pueden enseñar y aprender a lo largo de todo el ciclo vital. Tienen un efecto incremental, de forma que el desarrollo de una fortaleza favorece el desarrollo de otras. Según esta autora, una característica que distingue al ser humano es el impulso a desarrollar fortalezas para sentirse competente y satisfacer necesidades de pertenencia, afiliación y seguridad.

Las fortalezas son tanto internas como externas e interactúan entre sí; la integración de atributos individuales y contextuales promueve el desarrollo frente al riesgo. Como lo plantean Wright y López (2002), las dificultades y déficits de la persona y su contexto, es decir, los riesgos, deben enfrentarse con las áreas individuales de fortaleza, junto con los recursos externos de que se dispone.

La edificación de fortalezas tiene gran influencia en los procesos de desarrollo en todas sus dimensiones. De acuerdo con Scheel et al. (2013), las fortalezas aumentan la motivación para el cambio, ayudan a concretar metas, promueven la confianza en las propias capacidades para enfrentar dificultades y se pueden transferir de un ámbito de funcionamiento a otros.

La Orientación centrada en fortalezas se refiere a poner en marcha recursos y habilidades para resolver problemas y alcanzar metas, en concordancia con lo que indica el Ministerio de Educación Pública de Costa Rica (MEP, 2017), lo que ubica a la persona profesional en Orientación en una posición única en el acompańamiento del estudiantado en su desarrollo integral.

La identificación y utilización de fortalezas por parte del estudiantado es esencial para el logro del máximo potencial, el bienestar personal-social, el desarrollo vocacional y el logro académico. Sin embargo, esto no significa que la Orientación centrada en fortalezas 
aborde solamente lo positivo; es más bien una perspectiva de las posibilidades, es edificar con base en la potencialidad.

Si bien la Orientación centrada en fortalezas incluye elementos que han estado presentes en el quehacer de la disciplina desde sus inicios, resultan pertinentes las recomendaciones de Smith (2006) con respecto a lo que se debe resaltar en todas las intervenciones de este grupo profesional:

- Lo que la persona orientada tiene, en lugar de lo que no tiene.

- Los atributos personales utilizados en la resolución exitosa de diversos eventos.

- La validación de los esfuerzos que hace la persona en variedad de situaciones.

- el reconocimiento de contribuciones y aportes.

- El establecimiento de metas realistas o cambios deseados que fortalezcan la esperanza y aporten cambios concretos de acción.

- Las fuentes de donde se derivan la valía personal y el sentido de propósito.

- Los componentes económicos y socioculturales de los diversos eventos, lo que necesariamente implica activar recursos propios y externos.

De acuerdo con Zimmerman et al. (2013), la integración de fortalezas internas y externas es clave en las intervenciones de Orientación. Además, es pertinente incrementar las oportunidades para que el estudiantado aplique aspectos cognitivos y conductuales para reforzar habilidades que favorecen el enfrentamiento de la adversidad. Las intervenciones que estos autores recomiendan — desde la perspectiva de las fortalezas y con miras a la mitigación de los efectos perjudiciales de la exposición a adversidades - pueden ser compensatorias, tales como una fuerte identidad ante la presencia de estereotipos o prejuicios; protectoras, como las experiencias de éxito; o de desafio, que consisten en exposiciones paulatinas a dificultades.
Otra razón para enfatizar en las fortalezas es que su utilización incrementa los logros y el bienestar interno, los cuales tienen una influencia mutua: el bienestar interno favorece la obtención de logros y los logros favorecen el bienestar interno; a su vez, un bajo bienestar interno dificulta el alcance de logros y el contar con escasos logros perjudica el bienestar interno de las personas. La oportunidad para obtener logros en diferentes ámbitos es fundamental, porque permite identificar fortalezas y facilita el desarrollo de otras, lo cual beneficia la autoestima. En relación con esto, Bandura (1999) aportó el constructo de autoeficacia, una fortaleza interna que conceptualiza como autoestima y que se torna auténtica al poner en uso las propias capacidades durante la vivencia de diversas situaciones.

Con respecto a las fortalezas externas, una de las de mayor peso es el apoyo de personas adultas, tanto de parientes (no necesariamente los progenitores), como extrafamiliares. El apoyo de personas adultas ha mostrado tener efectos positivos en muchos retos, tales como autocuidado en la vida sexual, disminución de conductas violentas y uso de drogas, enfrentamiento de influencia negativa de pares y cumplimiento con lo requerido para rendir adecuadamente en el colegio (Zimmerman et al., 2013).

Como se verá en el siguiente apartado, hay una estrecha relación entre las fortalezas y los pilares de la resiliencia. Esto se debe a que la resiliencia, según Yates et al. (2008), se alcanza cuando se moderan los efectos de los riesgos y las personas desarrollan su competencia, entendida como el uso adaptativo de recursos tanto internos como externos, al enfrentar una adversidad.

\subsection{El proceso de resiliencia}

La resiliencia puede definirse de varias formas. Para Benard y Marshall (1997), es el proceso de desarrollo mediante el cual las personas satisfacen las necesidades esenciales de afecto, conexión, respeto, desafío, estructura, involucramiento significativo, pertenencia y poder. Las autoras conciben la resiliencia como sinónimo de empoderamiento y plantean que las condiciones para su activación son las mismas que 
las que promueven el aprendizaje y el desarrollo exitosos.

El Search Institute (2006) ha denominado esas condiciones "Elementos fundamentales del desarrollo", $y$ ha identificado veinte elementos internos y veinte externos sobre los que se sustenta la resiliencia. El bienestar y el desarrollo a futuro como personas adultas responsables son más factibles con la presencia de al menos tres cuartas partes de los elementos en la vida de las niñas, niños y jóvenes. Las personas que cuentan con menor cantidad de elementos son las que se encuentran en situación de mayor riesgo.

De acuerdo con Rutter (1993), pionero en el estudio acerca de este fenómeno, es un conjunto de mecanismos (no características aisladas) mediante los cuales las personas incrementan su capacidad para enfrentar el estrés, las dificultades y los riesgos. Algunos de estos mecanismos son la autoestima, la obtención de logros concretos y la creación de oportunidades.

Para Masten (2014), otra pionera que ha enriquecido el acervo de conocimiento al respecto, la resiliencia se entiende como el desarrollo positivo de una persona o sistema durante y después de dificultades significativas. Resalta que son los mecanismos humanos de adaptación los que hacen posible esa "magia de lo ordinario".

Según Zimmerman et al. (2013), es la interacción de factores individuales y contextuales, antes y durante eventos estresantes o situaciones de riesgo. Para que se dé un fortalecimiento, la interacción debe darse durante periodos largos de tiempo, por lo que Yates et al. (2008) son categóricos al afirmar que la resiliencia va mucho más allá de la persona y que refleja un proceso sostenido. Explican que, si se alcanza en una etapa del desarrollo, la persona queda con las bases para el logro y la resolución de problemas en periodos subsiguientes.

Las condiciones para la activación de la resiliencia — que, como se indicó (Benard \& Marshall, 1997), son las mismas que las que promueven el aprendizaje y el desarrollo exitosos - también se denominan pilares; son seis y se han clasificado en dos grupos. El primer grupo, relativo a la Mitigación de los riesgos, se compone de tres pilares: Enriquecimiento de vínculos prosociales, Establecimiento de límites claros y firmes y Aprendizaje de habilidades para la vida. El segundo grupo, denominado Construir resiliencia, aporta tres pilares adicionales, que son: Disfrute de forma constante de relaciones de afecto y apoyo, Transmisión de expectativas elevadas y Presencia de oportunidades para la participación significativa (Henderson \& Milstein, 2003).

Los estudios revisados sugieren una interrelación entre los pilares. Con base en la información que suministra el Instituto Search (2006) acerca de los elementos para un desarrollo satisfactorio de niñas, niños y adolescentes, en la Figura 1 se ilustra dicha interrelación entre los pilares del modelo de Henderson y Milstein (2003). Estos autores distinguen dos grupos de pilares: los que mitigan los riesgos y los que construyen resiliencia. Los que mitigan los riesgos, también llamados protectores, se muestran en la columna de fondo azul. Por su parte, los pilares para la construcción de la resiliencia, denominados promotores, se encuentran colocados en la columna de fondo amarillo. La interrelación entre los dos grupos de pilares se ilustra tomando como referencia los aportes del Search Instituto (2006) con respecto a los elementos de desarrollo, en filas de color verde, para indicar la interrelación de los colores utilizados para los grupos de pilares. 
Figura 1. Interrelación entre pilares para la mitigación de los riesgos y pilares que construyen resiliencia

\begin{tabular}{ll}
\hline Pilares para la mitigación de riesgos (protección) & \multicolumn{1}{c}{$\begin{array}{c}\text { Pilares para la construcción de resiliencia } \\
\text { (promoción) }\end{array}$} \\
\hline $\begin{array}{l}\text { 1. Enriquecimiento de los vínculos prosociales. Cone- } \\
\text { xiones con pares y personas adultas que promueven } \\
\text { la resiliencia y acceso a actividades constructivas. }\end{array}$ & $\begin{array}{l}\text { 4. Disfrute de forma sostenida de relaciones de afecto } \\
\text { y apoyo. Aceptación incondicional y presencia de per- } \\
\text { sonas confiables que pueden ayudar. }\end{array}$ \\
\hline
\end{tabular}

Personas adultas significativas que son afectuosas y tienen un comportamiento positivo. Amistades que son buen ejemplo de comportamiento responsable. Presencia de valores positivos como igualdad, justicia social, honestidad y responsabilidad. Empatía y sensibilidad en las relaciones interpersonales. Alteridad o reconocimiento de valía de la otra y el otro.

2. Establecimiento de límites claros y firmes. Normas y procedimientos que son claramente comunicados, en cuya implementación se evitan la incoherencia, la laxitud y la arbitrariedad.
5. Transmisión de expectativas elevadas. Expectativas motivadoras por ser altas pero realistas. Incluyen evitar el uso de etiquetas que conducen a no esperar logros por parte del estudiantado.

Familia e institución supervisan las actividades y tienen reglas y consecuencias claras. Motivación para rendir en los estudios y disposición a cumplir con las tareas escolares. Resistencia a la presión negativa por parte de pares. Seguridad en el hogar, la escuela y el vecindario.

3. Aprendizaje de habilidades para la vida. Incluye el aprendizaje de la cooperación, la resolución de conflictos, la asertividad y el manejo del estrés.
6. Presencia de oportunidad para la participación significativa. Acceso a toma de decisiones, establecimiento de metas, ejercicio del propio poder y apoyo a otras personas.

Implicación en actividades artísticas, deportivas, comunales y programas juveniles. Habilidad para planear asumir responsabilidad por las propias acciones. Convivencia respetuosa con personas de diferente marco cultural o étnico. Oportunidades para brindar servicios a la comunidad. Creación de ambientes que facilitan el aprendizaje

Nota: Elaboración propia con base en Henderson y Milstein (2003) y Search Institute (2006).

Debido a la relación de la resiliencia con los procesos de desarrollo en general, los seis pilares también se han asociado específicamente con las tareas del desarrollo psicosocial de Erikson (1985. Citado por Grotberg, 2006). Así, por ejemplo, el establecimiento de límites se ha asociado con el logro de la confianza, las habilidades sociales con la autonomía, el amor incondicional con la iniciativa y las oportunidades para la participación con la identidad.

La institución educativa es un espacio óptimo para promover los pilares de la resiliencia, los cuales implican la creación de estructuras que proporcionen seguridad, libertad para crecer y oportunidades para aportar y convivir de forma armónica (Acevedo \& Mondragón, 2005).

\section{Retos que más frecuentemente enfrenta el estudiantado}

La teoría acerca de las fortalezas humanas y la resiliencia aporta lineamientos para intervenciones preventivas en instituciones de educación primaria y secundaria, con el estudiantado que enfrenta uno o más obstáculos.

Es nuestra responsabilidad, desde la Educación en general y desde la Orientación en particular, la promoción de una visión respetuosa y equilibrada de las niñas, niños y jóvenes. Esto significa, de acuerdo con Marshak et al. (2010), que sus fortalezas no queden ensombrecidas por la presencia de dificultades. Se requiere una visión amplia y la máxima reducción de obstáculos en aquellos estudiantes que parecen no ajustarse a la escuela; se requiere, asimismo, evitar las 
distorsiones que magnifican los déficits, minimizan las fortalezas y no construyen un optimismo realista.

La aplicación del enfoque de fortalezas para construir resiliencia en el ámbito educativo es mencionada por Shapiro y Lebuffe (2006), como forma de preparación para enfrentar la adversidad y de disminuir problemas disciplinarios en el estudiantado y referencias a servicios de apoyo.

En relación con el ámbito latinoamericano, García-Vesga y Domínguez (2013) también aportan conocimientos acerca de la potenciación de fortalezas en la promoción de una adaptación resiliente por parte de estudiantes, lo que consideran aporta una mirada esperanzadora, que se aplica a contextos culturales diferentes. Los autores rescatan la importancia del entorno, en interacción continua con la persona, y reportan efectos en los comportamientos para la organización, la responsabilidad y los contactos sociales positivos; su trabajo se refiere a programas que se han desarrollado en Perú, Argentina y Brasil. Al igual que Henderson y Milstein (2003), destacan la pertinencia de favorecer los vínculos afectivos de apoyo de las personas menores con adultas y adultos de sus contextos educativos o familiares, así como la importancia de la participación en actividades constructivas.

En una investigación realizada en colegios nocturnos de Costa Rica con personas adultas jóvenes que no completaron la educación secundaria durante la adolescencia, se identificaron dificultades en los ámbitos académico, conductual y familiar, en este último, en lo relativo a situaciones de conflicto, enfermedad, adicción o pérdida (Arguedas-Negrini, 2019). Con base en consideraciones teóricas sobre estos temas, a continuación se presenta información específica acerca de los retos que más frecuentemente enfrenta el estudiantado de escuelas y colegios, así como recomendaciones para su abordaje desde la perspectiva de las fortalezas.

\subsection{Dificultades académicas}

Los autoconceptos académicos positivos son esenciales en la vida escolar; no implican negar las áreas de dificultad, sino más bien una visión equilibrada del conjunto de características relacionadas con el aprendizaje. Docentes y estudiantes necesitan saber que es posible enfrentar varias dificultades simultáneamente, y que estas interactúan con las fortalezas internas y externas. El autoconcepto académico está fuertemente influido por la realimentación que brindan las personas adultas, por lo que son indispensables los mensajes optimistas en relación con las posibilidades de mejorar. Para quienes enfrentan dificultades en el ámbito académico, es esencial no calificar las dificultades como algo permanente y que refleja baja capacidad de la persona, sino como retos particulares en áreas específicas. También hay que hacer referencia constante a la relación de las actividades escolares con la vida cotidiana y el mundo laboral (Marshak et al., 2010).

La experiencia escolar positiva ha mostrado ser una influencia protectora a lo largo del desarrollo. El estudiantado necesita múltiples experiencias de logro, el fomento de la motivación intrínseca y la autoeficacia y persistencia ante las dificultades (Masten et al., 2008).

Acevedo y Mondragón (2005) se refieren a la resiliencia escolar como un proceso que va más allá del rendimiento académico y se dirige también a la formación de la conciencia cívica y a los legados culturales e históricos de su región. Resaltan los efectos positivos de ver a las estudiantes y los estudiantes como participantes activos y no como receptores pasivos, "como valiosos recursos en lugar de usuarios de recursos" (p. 30), con altas posibilidades de superación.

Para niñas, niños y adolescentes, el apoyo de personas adultas que les consideran capaces como estudiantes y valiosos como personas es especialmente decisivo para la edificación de la confianza, la autovaloración y la reciprocidad social. A esto se unen los avances en neurodesarrollo que explican los efectos de las experiencias tempranas en el desarrollo posterior (Yates et al., 2008). 


\subsection{Problemas conductuales}

El apoyo de la institución es esencial y está representado en múltiples acciones que fomentan el gusto por aprender y en la provisión de oportunidades para desarrollar responsabilidad personal e interpersonal. Además del apoyo al estudiantado que no está teniendo logro académico, es necesaria la atención de quienes no logran mantener relaciones con sus pares o que tienen problemas conductuales en el aula, porque, de continuar esas dificultades, se pone en riesgo su trayectoria vital. Milsom y Glanville (2010) se refieren a la relación de las habilidades sociales con el rendimiento del estudiantado, especialmente la cooperación, la asertividad y el autocontrol.

Las llamadas funciones ejecutivas son determinantes en el desarrollo de habilidades, ya que, según Monge (2016), son las actividades mentales que le permiten a la persona llevar a cabo tareas de manera intencional; consisten en la aplicación del pensamiento para resolver problemas o implementar planes que llevan al logro de metas; en otras palabras, transformar el pensamiento en acciones. Las funciones ejecutivas permiten autorregular el comportamiento. Algunos ejemplos son la anticipación, el control atencional, el automonitoreo y el control de las emociones propias.

Si bien las funciones ejecutivas son capacidades cognitivas, tienen efectos tanto en el rendimiento académico como en el comportamiento, porque son las que permiten reaccionar de manera adecuada ante situaciones, usando procesos mentales como la autorregulación en el manejo de las reacciones emocionales (Ramos-Galarza et al., 2017). Otras autoras que han encontrado una relación entre las funciones ejecutivas y los problemas de conducta son Romero et al. (2016).

De acuerdo con Bertella et al. (2018), el tema de las funciones ejecutivas es de especial importancia cuando las familias de las niñas, niños o adolescentes se encuentran en situaciones de riesgo social; es frecuente que los riesgos se presenten en conjunto con condiciones de pobreza, pero no sucede así en todos los casos. El riesgo corresponde a contextos en los que se ve afectada la satisfacción de necesidades que conducen al bienestar y a la calidad de vida, lo que deja a sus integrantes, especialmente las personas menores, en desventaja para el logro de sus tareas del desarrollo correspondientes a su edad. Las autoras agregan que, a nivel escolar, las funciones ejecutivas tienen efectos en el aprendizaje y en la regulación de la propia actividad, ya que favorecen la autodirección para posponer la gratificación y cumplir diversas tareas con las correspondientes etapas de iniciación, planificación y adopción de comportamientos estratégicos.

En relación con personas menores que pertenecen a familias pobres, Elliott y Elliott (2011) alertan acerca de las implicaciones negativas de la presencia de múltiples factores de riesgo que pueden ser acumulativos. En condiciones de pobreza hay mayor incidencia de elevados niveles de estrés en la familia, insuficiente nutrición, bajo nivel educativo y depresión, todo lo cual hace que los mecanismos para la adopción de comportamientos autorregulados sean inestables o erráticos.

\subsection{Situaciones familiares}

Otra adversidad que enfrentan niñas, niños y adolescentes es la que se origina en situaciones familiares. Es frecuente que sufran violencia en el hogar, de forma directa o como testigos. Elliott y Elliott (2011) plantean que las manifestaciones que tienen niñas, niños y jóvenes que han sido víctimas o han observado violencia en el hogar presentan bastante similitud con los indicadores físicos, emocionales y conductuales de personas agredidas físicamente. Las respuestas emocionales al abuso giran alrededor de la culpa, la vergüenza, el sentido de responsabilidad y la desesperanza por lo que ocurre en el hogar. Algunas manifestaciones conductuales son agresividad o su polo opuesto, pasividad, inasistencia a la institución, bajo rendimiento e intentos de suicidio. Los indicadores físicos incluyen cansancio, higiene deficiente, uso de sustancias, trastornos psicosomáticos y conductas de alto riesgo. Ante eventos de este tipo, es esencial la activación de los pilares para la mitigación de los riesgos y para la construcción de la resiliencia. Es importante tomar esto en cuenta, 
ya que esas manifestaciones de privación y angustia pueden confundirse con desinterés hacia el colegio o indisciplina.

En los casos de situaciones familiares que implican desatender las necesidades de las personas menores, puede estar indicada la referencia a instancias externas e incluso la activación de protocolos. No obstante, es relevante también el apoyo para la identificación de formas constructivas de responder a la adversidad, de acuerdo con la concepción de Duarte y Cardoso (2014) de que el ser humano, más que actor de su existencia, es autor. Sin ignorar la desigualdad, el abuso y los obstáculos, los significados que se adscriben a las diferentes circunstancias pueden modificarse, acercándolos al optimismo y la esperanza, mediante la identificación de lecciones aprendidas, acciones encaminadas a logros concretos e identidad basada en fortalezas.

\section{Conclusiones}

Para contrarrestar serias dificultades que a menudo sufre la población estudiantil, los conocimientos disponibles deben conectarse con la multiplicidad de acciones que se implementan desde las instituciones educativas para el beneficio del estudiantado. También debe existir una conexión del personal docente y administrativo con su propia resiliencia, para así modelarla al estudiantado.

La persona profesional en Orientación está en una posición central para promover el bienestar integral de la niñez y la adolescencia. La edificación de fortalezas es un componente de primordial importancia en el abordaje de los procesos socioeducativos en instituciones de educación primaria y secundaria por su contribución a ese doble papel de la institución educativa de promover el desarrollo y proteger de la adversidad a la totalidad del estudiantado. Se cuenta con numerosas experiencias que han mostrado que arreglar problemas es mucho menos efectivo que promover el desarrollo.

A la vez, es innegable que quienes están en las etapas de la nińez y la adolescencia están expuestos a situaciones de riesgo. Si los riesgos son múltiples, las protecciones y los apoyos tienen que ser mayores. Se recomienda que las escuelas y colegios integren la mayor cantidad posible de componentes de los pilares, tanto de mitigación de riesgos como de construcción de resiliencia. Es pertinente también revisar las concepciones acerca del estudiantado que tiene dificultades, para cuestionar estereotipos, así como ofrecer oportunidades y transmitir la creencia en la capacidad de transformación.

Las niñas, niños y jóvenes merecen desenvolverse en un ambiente escolar nutricio que favorezca los pilares del fortalecimiento, los cuales, en su conjunto, construyen esperanza. Las personas profesionales en Orientación se encuentran en una posición idónea para influir positivamente en este proceso.

\section{Agradecimientos}

La autora agradece su apoyo a la Escuela de Orientación y Educación Especial y al Instituto de Investigación en Educación, ambos de la Facultad de Educación, Universidad de Costa Rica. El trabajo se elaboró dentro del proyecto N. ${ }^{\circ}$ 724-B7-304, que recibió el financiamiento de la Vicerrectoría de Investigación de la institución.

\section{Referencias bibliográficas}

Acevedo, V. E., \& H. Mondragón, H. (2005). Resiliencia y escuela. Pensamiento Psicológico, 1(5), 21 35. http://r.issu.edu.do/l.php?l=165DRe

Arguedas-Negrini, I. (2019). Autorrealización en personas adultas jóvenes: fuentes y estrategias para su construcción. Revista de Educación y Desarrollo, (51), 9-18. http://r.issu.edu.do/l.php?l=89viJ

Bandura, A. (1999). Auto-eficacia: cómo afrontamos los cambios de la sociedad actual. Bilbao: Desclée de Brouwer.

Benard, B., \& Marshall, K. (1997). A Framework for Practice: Tapping Innate Resilience. University of Minnesota: The Center for Applied Research and Educational Improvement.

Bertella, M. A., Grebe, M. P., Dalbosco, M. S., \& Al- 
ba-Ferrara, L. (2018). Funciones ejecutivas, pobreza y estimulación cognitiva: un andamiaje para futuras intervenciones. Avances En Psicología, 26(1), 33-40. https://doi.org/10.33539/avpsicol.2018.v26n2.1125

Duarte, M. E., \& Cardoso, P. (2014). The life design paradigm: from practice to theory. En L. Nota \& J. Rossier (Eds.), Handbook of Life Design: From Practice to Theory and from Theory to practice (pp. 41-57). Gotinga, Alemania: Hogrefe.

http://r.issu.edu.do/l.php?l=166C7c

Elliott, K. W., \& Elliott, J. K. (2011). Childhood observers of domestic violence. Alexandria, EEUU: American School Counselor Association. http://r.issu. edu.do/l.php?l=167l3a

García-Vesga, M. C., \& Domínguez, E. (2013). Desarrollo teórico de la resiliencia y su aplicación en situaciones adversas: Una revisión analítica. Revista Latinoamericana de Ciencias Sociales, Niñez y Juventud, 11(1), 63-77. http://r.issu.edu.do/l.php?l=168XVx

González-Arratia, N. I., Valdez, J. L., Oudhof, H., \& González, S. (2009). Resiliencia y salud en niños y adolescentes. Ciencia Ergo Sum, 16(3), 247-253. http://r.issu.edu.do/l.php?l=164zE1

Grotberg, E. (2006). ¿Qué entendemos por resiliencia? ¿Cómo promoverla? ¿Cómo utilizarla? En E. Grotberg (Ed.), La resiliencia en el mundo de hoy. Cómo superar las adversidades (pp. 17-57). Barcelona: Gedisa, S. A.

Henderson, N., \& Milstein, M. (2003). Resiliencia en la escuela. Buenos Aires: Paidós.

Marshak, L. E., Dandeneau, C. J., Prezant, F. P., \& L'Amoreaux, N. A. (2010). The School Counselor's Guide to Helping Students with Disabilities. San Francisco, California: Jossey-Bass Teacher.

Masten, A. (2011). Resilience in children threatened by extreme adversity: Frameworks for research, practice, and translational synergy. Development and Psychopathology, 2(23), 493-506.

https://www.doi.org/10.1017/S0954579411000198.
Masten, A. (2014). Ordinary Magic. Resilience in Development. New York: The Guilford Press.

Masten, A. Herbers, J. E., Cutuli, J. J., \& Lafavor, T. L. (2008). Promoting competence and resilience in the school context. Professional School Counseling, 12(2), 76-84.

https://www.doi.org/10.1177/2156759X0801200213

Milsom, A., \& Glanville, J. (2010). Factors mediating the relationship between social skills and grades in a sample of students diagnosed with learning disabilities or emotional disturbance. Remedial and Special Education, 31(4), 241-251.

https://www.doi.org/10.1177/0741932508327460

Ministerio de Educación Pública. (2017). Educar para una Nueva Ciudadanía. Programas de Estudio de Orientación Primero, Segundo y Tercer Ciclos de la Educación General Básica y Educación Diversificada. San José, Costa Rica. http://r.issu.edu.do/l.php?l=169K2G

Monge, M. (2016). Aprendizaje, cognición y neurociencia. San José, Costa Rica: EUNED.

Murillo, J., Martínez, C., \& Hernández, R. (2011). Decálogo para una Enseñanza Eficaz. REICE. Revista Iberoamericana sobre Calidad, Eficacia y Cambio en Educación, 9(1), 6-27.

http://hdl.handle.net/123456789/1537

Ramos-Galarza, C., Jadán-Guerrero, J. Paredes-Núñez, L., Bolaños-Pasquel, M., Santillán-Marroquín, W., \& Pérez-Salas, C. (2017). Funciones ejecutivas y conducta de estudiantes secundarios ecuatorianos. Revista Mexicana de Neurociencia, 18(6), 32-40. http://r.issu.edu.do/l.php?l=170fAb

Romero, M., Benavides, A., Quesada, A. B., \& Álvarez, G. (2016). Problemas de conducta y funciones ejecutivas en niños y niñas de 5 años. International Journal of Developmental and Educational Psychology, 1(1), 57-66.

https://www.doi.org/10.17060/ijodaep.2016.n1.v1.214

Rutter, M. (1993). La "Resiliencia": Consideraciones conceptuales. Journal of Adolescent Health, 14(8), 690-696. 
https://www.doi.org/10.1016/1054-139X(93)90196-V

Scheel, M. J., Davis, C. K., \& Henderson, J. D. (2013). Therapist Use of Client Strengths: A Qualitative Study of Positive Processes. The Counseling Psychologist, 41(3), 392-427.

https://www.doi.org/10.1177/0011000012439427

Search Institute. (2006). 40 Elementos fundamentales del desarrollo. http://r.issu.edu.do/l.php?l=171u4W

Shapiro, V. B., \& Lebuffe, P. A. (2006). Using Protective Factors in Practice. Lessons Learned about Resilience from a Study of Children Aged Five to Thirteen. Annals of the New York Academy of Sciencies, 1094(1), 350-353.

http://r.issu.edu.do/l.php?l=172bJR

Smith, E. J. (2006). The Strength-based counseling model. The Counseling Psychologist, 34(1), 13-79. https://www.doi.org/10.1177/0011000005277018
Wright, B. A., \& López, S. J. (2002). Widening the diagnostic focus: A case for including human strengths and environmental resources. En C. R. Snyder \& S. J. López (Eds.), Oxford Handbook of positive psychology (pp. 26-44). New York: Oxford University Press.

https://www.doi.org/10.1093/oxford$\mathrm{hb} / 9780195187243.013 .0008$

Yates, T. M., Egeland, B., \& Sroufe, A. (2008). Rethinking resilience: A developmental process perspective. En S. S. Luthar (Ed.), Resilience and Vulnerability: Adaptation in the Context of Childhood Adversities (pp. 243-266). New York: Cambridge University Press.

Zimmerman, M. A., Stoddard, S. A., Eisman, A. B., Caldwell, C. H., Aiyer, S. M., \& Miller, A. (2013). Adolescent resilience: Promotive factors that inform prevention. Child Development Perspectives, 7(4), 215-220. https://www.doi.org/10.1111/cdep.12042

\section{CÓMO CITAR:}

Arguedas-Negrini, I. (2020). Intervenciones de orientación para edificar fortalezas en niñas, niños y adolescentes. Revista Caribeña de Investigación Educativa (RECIE), 4(2), 127-136. https://doi.org/10.32541/recie.2020.v4i2.pp127-136 